\title{
BEYOND VIRTUAL CRYSTAL AND MOLECULAR FIELD APPROXIMATIONS IN DILUTED MAGNETIC SEMICONDUCTORS
}

\author{
J. TWORZYDLO \\ Institute of Theoretical Physics, Warsaw University \\ Hoża 69, 00-681 Warszawa, Poland
}

The framework of the virtual crystal approximation and the molecular field approximation has been widely used in the description of the extended states affected by the presence of the $p-d$ exchange interaction in diluted magnetic semiconductors. We review shortly the known examples of diluted magnetic semiconductors systems which are described beyond these approximations. The interesting case constitutes the nearly binding impurity potential in $\mathrm{Cd}_{1-x} \mathrm{Mn}_{x} \mathrm{~S}$. The lattice approaches developed earlier allowed to prove in this case the breakdown of the virtual crystal approximation for low concentrations of magnetic ions and to explain the apparent concentration dependence of the exchange integral. We take explicitly into account the substitutional disorder neglected in the lattice theories and we rederive these results within the dilute and the average $t$-matrix alloy approach. We generalize systematically this theory to include also the magnetic disorder of the dilute spin subsystem appearing at finite temperatures. When the off-diagonal exchange scattering terms are taken into account in our calculations, we get a good agreement with the experimental data on the asymmetric field dependent spin splitting and the zero-field concentration dependence of the energy gap.

PACS numbers: 71.55.Gs, 75.50.Pp

\section{Introduction}

The standard virtual crystal (VC) and the molecular field (MF) approximations [1] have been extensively and successfully used in the interpretation of magneto-optical experiments in diluted magnetic semiconductors (DMS). These approaches allowed to determine precisely the magnitude of exchange integrals for numerous materials [2]. The microscopic theory of the $s p-d$ exchange coupling has also been developed leading to the further progress in understanding the physical origin of the magnetic interactions and the chemical trends in DMS [3].

It is generally accepted that the $\mathrm{VC}$ and the MF approximations are appropriate if the magnitude of the exchange integral and the chemical substitutional 
potential are small with respect to the relevant bandwidth (the weak coupling case). For the localized states the effective bandwidth tends to zero and thus these approximations are not applicable (the strong coupling case). Indeed, the magnetic polaron effect [4], present in DMS only for states localized by impurities or alloy fluctuations, was treated in formalisms appropriate for the opposite, strong coupling limit. The magnetically interacting localized complexes observed in some II-VI compounds with $\mathrm{Cu}, \mathrm{Ni}$ impurities [5] and also the $\mathrm{ZnS}: \mathrm{Mn}[6]$ and the GaAs:Mn [7] systems belong to this class of problems.

In this paper we concentrate, however, on the problem of the influence of magnetic impurities upon the extended electronic states only. One of the manifestations of this influence is the peculiar dependence of the energy gap on the temperature in certain investigated DMS [8]. The magnetic contributions to this dependence were described by means of the second order perturbation corrections to the MFA [9]. Some features of an anomalous Zeeman splitting at high concentrations were also derived in this framework [10].

The most spectacular manifestation of the kinetic exchange interaction is the field induced spin splitting. The challenging question seems to be a possibility to trace the evolution of this splitting from the weak coupling (where the VCA and the MFA applies) to the strong coupling case. It is evident that for the exchange interaction strong enough the extension of not only the MFA but also of the VCA should be examined.

The breakdown of the VCA and the MFA is expected to be manifested experimentally by the concentration and temperature dependence of the exchange integrals. The experimental data for IV-VI DMS compounds [11] seem to exhibit such dependence. The interpretation of these results [12] is however overshadowed by the complexity of the band structure and the nonisovalent character of the magnetic impurities.

The very striking example of the breakdown of the VCA constitutes the anomalous concentration dependence of the exchange integrals found in $\mathrm{Cd}_{1-x} \mathrm{Mn}_{x} \mathrm{~S}$ [13-16]. The value $\beta N_{0} \approx-2 \mathrm{eV}$ of the exchange constant for the $A$ valence band was reported $[15,16]$ for $x \approx 1.5 \%$, the highest concentration that allowed experimental investigations of the Zeeman splitting in $\mathrm{Cd}_{1-x} \mathrm{Mn}_{x} \mathrm{~S}$. The samples with small concentration of magnetic impurities $x \approx 0.1 \%[13,15]$ shows a substantial enhancement of this constant: $\beta N_{0} \approx-5 \mathrm{eV}$. The magnitude of the exchange integrals inferred from experiments remained in disagreement with the local spin density approximation (LSDA) calculations and with the chemical trends predictions [3]. The asymmetric spin splitting of the $\sigma^{-}, \sigma^{+}$components $[13,14]$ of the $A$-exciton as a function of the external magnetic field constitutes other anomaly, which cannot be interpreted within the VCA and the MFA. The collection of the experimental data [13-18] on the zero field dependence of the energy gap on the concentration $x$ shows a nonmonotonic behaviour, indicating the presence of the "bowing" effect for small $x$ values. Both the VCA and the effect of nonmagnetic alloy corrections [17] fail in the interpretation of this bowing.

The first quantitative explanation of a part of the above observations was proposed by Benoit à la Guillaume et al. [19]. The authors suggested the particularly strong exchange interaction in $\mathrm{Cd}_{1-x} \mathrm{Mn}_{x} \mathrm{~S}$ and large hole effective mass 
to be responsible for a nearly binding substitutional potential of $\mathrm{Mn}$ ions. To circumvent the VCA in the description of the Zeeman splitting they introduced an intuitive model, which allowed to calculate the energy shifts by the Wigner-Seitz approach (WS). The Mn ions were described by a simple model potential with some free parameters and were placed in the saturating external magnetic field. In order to apply the WS method, the magnetic ions were arranged in an artificial superlattice with the concentration dependent period.

In the subsequent publication [20] this approach was generalized to calculate also the zero field variation of the band gap. Firstly, the results obtained at saturation with the use of the WS method were checked by the plain wave expansion (PWE) calculations for different shape of the Mn ion potential. Secondly, the system at zero magnetic field was described by considering an elementary cell of the superlattice containing several classical spins with the zero total spin momentum. The effects of both the chemical and the magnetic disorder were thus disregarded.

\section{The alloy approach}

The main motivation of our work was to get rid of the artificial assumption of the periodic distribution. In order to include the chemical disorder one should make appeal to the alloy theory [21]. The physical principle of this theory states that only measurable quantities can be averaged over random distributions of impurities. The density of states, related to the Green function of the particle, is an example of such physical quantity. The averaging procedure is thus performed on the perturbation series for the Green function [22] and leads to the so-called multiscattering series [21]. The scattering events are collected into the irreducible self-energy $\Sigma_{k}(E)$. The averaged Green function is then related to $\Sigma$ with the use of the Dyson equation.

We are not interested in the expression for the whole density of states here. In our alloy approach the quantity we want to calculate is the configurationally and thermodynamically averaged position of the band ellge. The value of this energy $E_{0}$ is calculated [23] from the dispersion relation taken at $k=0$

$$
E_{0}+E_{k=0}+\Sigma_{k=0}\left(E_{0}\right)=0 \text {. }
$$

The analytic formula for $\Sigma_{k=0}(E)$ was calculated by the author in Ref. [23]. Similarly to Ref. [19] a square-well model potential of a $\mathrm{Mn}$ ion at saturation and one band effective mass approximation for holes were assumed. The obtained result was exact only up to linear terms in the concentration $x$, but was nonperturbative in the magnitude $U$ of the $\mathrm{Mn}$ ion potential. We denote here this result by $\Sigma_{0}(E ; U)$. In spite of the used dilute limit approximation (DLA), the calculated energy shift exhibited a nonlinear dependence on the concentration $x$ showing the clear discrepancy with the VCA.

The alloy theory approach as well as the lattice approaches were employed in the interpretation of the spin splitting in $\mathrm{Cd}_{1-x} \mathrm{Mn}_{x} \mathrm{~S}$ according to the procedure worked out in Ref. [19]. The valence band-offset was fixed at $W N_{0}=-0.5 \mathrm{eV}$ [19] and the averaged effective mass was assumed $m^{*}=1.35 m_{\mathrm{e}}[20]$. The exchange integral $\beta N_{0}$ and the radius $b$ of the Mn ion substitutional potential were fitted to a few existing experimental data and estimated as follows: 
1) $\beta N_{0}=-1.4 \mathrm{eV}, b=4.1 \AA$ in the alloy theory,

2) $\beta N_{0}=-1.5 \mathrm{eV}, b=3.5 \AA$ in the lattice theories.

We note that the alloy theory as well as the superlattice approaches proved to be quite accurate in the description of the $A$-exciton spin splitting in $\mathrm{Cd}_{1-x} \mathrm{Mn}_{x} \mathrm{~S}$ at saturation. The estimates of the exchange integral are in agreement with the LSDA calculations in MnS [3]. We conclude that the explanation of the apparent dependence of the exchange integral in $\mathrm{Cd}_{1-x} \mathrm{Mn}_{x} \mathrm{~S}$ on the concentration $x$ is possible only beyond the VCA, when the nearly binding substitutional potential of a $\mathrm{Mn}$ ion is taken into account in a nonperturbative way.

\section{The magnetic disorder}

The standard alloy theory does not include the magnetic disorder introduced by the presence of the spin fluctuations at the finite temperatures. To take it into account we used the Matsubara formalism of the imaginary time dependent Green functions [24]. We assume the localized spins of impurities, described by operators $\hat{S}_{i}$, to be independent and to interact only with the external magnetic field $H$. The spins are in thermal equilibrium with the crystal lattice. The holes interact with the spin subsystem via the Heisenberg-like exchange interaction and are also scattered by the spin-independent potential of magnetic impurities. One may derive the appropriate series for a temperature Green function of the particle interacting with the system of randomly distributed Mn ions. The series simplifies considerably when we assume the limit of a one particle placed in an empty band. The resulting perturbation expansion contains only the free particle propagators and correlation functions for the random spin subsystem. The correlation functions do not depend on the imaginary time in three specific cases:

1) for an Ising quantum spin $\widehat{\boldsymbol{S}}_{i}=\left(0,0, \widehat{S}_{i}^{z}\right)$,

2) for a classical vector $\operatorname{spin} S_{i}=S\left(\sin \theta_{i} \cos \phi_{i}, \sin \theta_{i} \sin \phi_{i}, \cos \theta_{i}\right)$,

3) for a quantum spin provided that the direct Zeeman term for band particle with the same $g$-factor was introduced.

The procedure of configurational averaging of the perturbation expansion becomes analogous to the calculations of the alloy theory at saturation. The simple vertex factors are replaced by combinations of the thermodynamical averages of spin matrices and powers of concentration $x$ [24]. The infinite summation of the series for the irreducible self-energy can be effectively conducted with the DLA (only terms linear in $x$ are included) or with the more sophisticated average $t$-matrix approximation (ATA). The results in the DLA are expressed for two spin polarizations $\sigma= \pm 1$ in a closed form:

1) for the Ising spin

$\Sigma_{\sigma}=\sum_{m=-S}^{S} p(m \sigma) \Sigma_{0}\left(E ; U_{m}\right)$

2) for the classical spin

$\Sigma_{\sigma}=\frac{1}{2}\left(1+\sigma \frac{\mu}{S}\right) \Sigma_{0}\left(E ; U_{S}\right)+\frac{1}{2}\left(1-\sigma \frac{\mu}{S}\right) \Sigma_{0}\left(E ; U_{-S}\right)$, 
3) and for the quantum spin

$$
\Sigma_{\sigma}=\frac{S}{2 S+1}\left[\left(1+\frac{1+\sigma \mu}{S}\right) \Sigma_{0}\left(E ; U_{S}\right)+\left(1-\frac{\sigma \mu}{S}\right) \Sigma_{0}\left(E ; U_{-S-1}\right)\right]
$$

where $p(m)$ is the Boltzmann distribution of spins with $m, \Sigma_{0}(E ; U)$ denotes the self-energy calculated for the one-impurity scattering problem and the potential magnitude is $U_{m}=-\frac{1}{2} m N_{0} \beta+W N_{0}$. We have introduced $\mu$, which is the dimensionless magnetization of one spin $\left(\mu=\left\langle S_{x}\right\rangle\right)$. To calculate $\Sigma$ with the ATA one needs only to rescale the potential magnitude by the concentration and to shift the reference energy by the MFA result [24].

The self-energy calculated for the quantum spin (4) was approximated by neglecting the direct Zeeman splitting of the particle energy. We note that the result (4) is qualitatively very similar to the result (3) and reduces to the classical spin result in the limit $S \rightarrow \infty$. The result (2) was also discussed in Ref. [23], but there it was derived from intuitive arguments only. The advantage of the results (3), (4) is that they take into account the processes of a carrier spin flip, which are expected to be important in the case $H=0$.

We remind that the spin dependent position of the band edge is calculated with the use of the self-consistent condition (1) for the self-energy (2), (3), (4). The calculations for the paramagnetic case, based on the results derived in the Matsubara formalism in the DLA, are depicted in Fig. 1. The parameters $\beta N_{0}$ and $b$ have been adopted from the fits to the spin splitting at the saturating magnetic field. We note that the corrections for the quantum spin represent only a small contribution to the calculated energy band dependence on $x$ for the classical

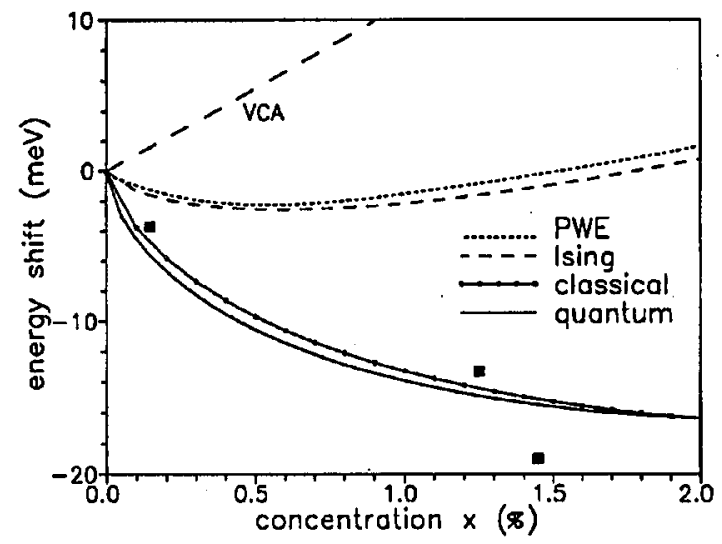

Fig. 1. The results for the energy gap dependence on the concentration $x$ for $H=0$. Experimental points () are taken from Refs. [12-15]. 
spin. We compare our results with that found by PWE for an artificial periodic structure. The experimental data are reproduced well when both the presence of magnetic disorder and the spin flip scattering are taken into account. This points out to the importance of these two effects at the zero external magnetic field.

The bowing effect in our model is temperature independent. This is the direct consequence of the independent spin model assumed in this work. The model proposed by Bylsma et al. in Ref. [8] ascribed the bowing effect in $\mathrm{Zn}_{1-x} \mathrm{Mn}_{x}$ Se only to the antiferromagnetic correlation between impurity spins. They found within the second order perturbation theory that the magnetic fluctuation corrections to the energy gap $\Delta E$ are proportional to the susceptibility: $\Delta E=-b T_{\chi}$. Their parameter $b$ fitted to the temperature dependence of the energy gap was however $\approx 4$ times greater than that needed to explain the concentration dependence. This disagreement clearly shows that not only the antiferromagnetic coupling but also the spin-dependent scattering is needed to explain the origin of the bowing effect in DMS.

With our alloy theory we can also calculate the dependence of the energy position of $\sigma^{-}, \sigma^{+}$components as a function of the external magnetic field, inaccessible in PWE calculations. The calculation of the spin splitting for the sample with the concentration $x=0.145 \%$ within the DLA leads to somewhat ambiguous results for the $\sigma^{-}$component since in a certain range of magnetic fields Eq. (1) has two solutions. For the more sophisticated approximation ATA we get a consistent theory in the whole range of magnetic fields and for concentrations up to a few percent.

We fitted the ATA alloy theory for the classical spin to the experimental data on the spin splitting at saturating magnetic field. The obtained parameters $b=4.3 \AA, \beta=-1.4 \mathrm{eV}$ are only slightly modified if compared with the DLA result. The energy gap dependence on the concentration at zero magnetic field was well

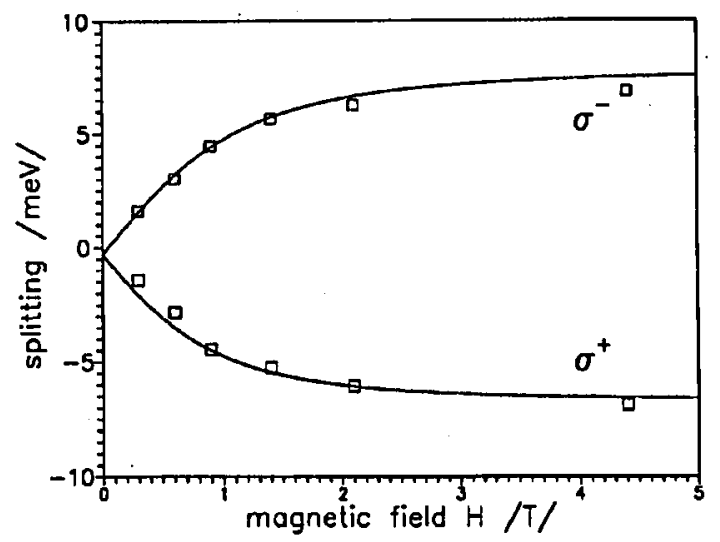

Fig. 2. The asymmetric spin splitting of the $A$-exciton in $\mathrm{Cd}_{1-x} \mathrm{Mn}_{x} \mathrm{~S}$ as a function of the magnetic field. Experimental points ( $\square$ ) were taken from Ref. [12], the solid line was calculated from the ATA theory with $\beta=-1.4 \mathrm{eV}, b=4.3 \AA$. 
reproduced too. We compare the experimental dependence [13] of the spin splitting on the external magnetic field with the predictions of the alloy theory in Fig. 2. The asymmetric spin splitting is excellently predicted by our model. In spite of the fact that the spin splitting exhibits a clearly non-Brillouin behaviour, we find that the total band spin splitting $\Delta E$ versus magnetization is linear with a good accuracy. The deviations of the ratio $\Delta E / \mu$ from the constant value for different magnetic fields amount to about $7 \%$ and are slightly smaller for higher concentrations $x$.

Summarizing, we emphasize that existing schemes of the alloy theory, possibly supplemented by the finite temperature formalism, offer a relatively simple and adequate description of the extended states beyond the VCA and MFA in low concentration DMS. The most important condition to observe the discrepancy with the VCA is the presence of the large $p-d$ exchange interaction and the large hole effective mass. It seems that these conditions are fulfilled for: $\mathrm{ZnS}$, CdS with $\mathrm{Fe}, \mathrm{Co}$ and $\mathrm{ZnSe}$ with $\mathrm{Fe}, \mathrm{Co}, \mathrm{Mn}$. The preliminary results indicate a presence of the spin-scattering corrections to the band gap bowing in CdFeS [25].

The author would like to acknowledge Professor J. Blinowski for many useful discussions and careful reading of the manuscript. The author acknowledges also the scholarship of Foundation for Polish Science.

\section{References}

[1] G. Bastard, C. Rigaux, Y. Guldner, J. Mycielski, A. Mycielski, J. Phys. (Paris) 39, 87 (1978).

[2] For a review see e.g. Diluted Magnetic Semiconductors, Eds. J.K. Furdyna, J. Kossut, in: Semiconductors and Semimetals, Vol. 25, Eds. R.K. Willard'son, A.C. Beer, Academic, Boston 1988; J.P. Lascaray, in: Semimagnetic Semiconductors and Diluted Magnetic Semiconductors, Eds. M. Averous, M. Balkanski, Plenum, London 1991, p. 169.

[3] K.C. Hass, in: Semimagnetic Semiconductors and Diluted Magnetic Semiconductors, Eds. M. Averous, M. Balkanski, Plenum, London 1991, p. 59; B.E. Larson, K.C. Haas, H. Ehrenreich, A.E. Carlson, Phys. Rev. B 37, 4137 (1988).

[4] T. Dietl, J. Spałek, Phys. Rev. Lett. 48, 355 (1982); J. Warnock, P.A. Wolf, Phys. Rev. $B$ 31, 6579 (1985).

[5] M. Godlewski, K. Świątek, A. Suchocki, J. Cryst. Growth 117, 634 (1992).

[6] H. Kawai, T. Hoshina, Solid State Commun. 22, 391 (1977).

[7] J. Schneider, U. Kaufman, W. Wilkening, M. Baeumler, F. Köhl, Phys. Rev. B 59, 240 (1987).

[8] J. Diouri, J.P. Lascaray, M. El Amrani, Phys. Rev. B 31, 7995 (1985); R.B. Bylsma, W.M. Becker, J. Kossut, U. Debska, D. Yoder-Short, Phys. Rev. B 33, 8207 (1986); J.A. Gaj, A. Golnik, Acta Phys. Pol. A 71, 197 (1987).

[9] A.K. Bhattacharjee, Solid State Commun. 65, 275 (1988); J.P. Lascaray, D. Coquillat, J. Deportes, A.K. Bhattacharjee, Phys. Rev. B 38, 7602 (1988).

[10] H. Pascher, P. Rothlein, G. Bauer, M. von Ortenberg, Phys. Rev. B 40, 10469 (1989).

[11] T. Dietl, C. Sliwa, G. Bauer, H. Pascher, Phys. Rev. B 49, 2230 (1994).

[12] S.I. Gubarev, Zh. Eksp. Teor. Fiz. 80, 1174 (1981). 
[13] G. Abrammishvili, S.I. Gubarev, A.V. Komarov, S.M. Rybchenko, Fiz. Tverd. Tela 26, 1095 (1984) [Sov. Phys.-Solid State 26, 666 (1984)].

[14] S.I. Gubarev, M.G. Tyazlov, Pis'ma Zh. Eksp. Teor. Fiz. 44, 385 (1986) [JETP Lett. 44, 494 (1986)]; 48, 437 (1988) [ 48, 481 (1988)]; Fiz. Tverd. Tela 32, 635 (1990) [Sov. Phys.-Solid State 32, 373 (1990)].

[15] M. Nawrocki, J.P. Lascaray, D. Coquillat, M. Demianiuk, in: Materials for Infrared Detectors and Sources, Eds. R.L. Aggarwal, J.K. Furdyna, S. von Molnar, MRS Symposia Proceedings No. 89, Materials Research Society, Pittsburgh 1987, p. 65.

[16] M. Ikeda, K. Itoh, H. Sato, J. Phys. Soc. Jap. 25, 455 (1968).

[17] O. Goede, W. Heimbrodt, M. Lamla, V. Weinhold, Phys. Status Solidi B 146, K65 (1988).

[18] C. Benoit à la Guillaume, D. Scalbert, T. Dietl, Phys. Rev. B 46, 9853 (1992).

[19] D. Scalbert, A. Ghazali, C. Benoit à la Guillaume, Phys. Rev. B 48, 17752 (1993).

[20] H. Ehrenreich, L.M. Schwartz, in: Solid State Physics, Vol. 31, Eds. H. Ehrenreich, F. Seitz, D. Turnbull, Academic Press, New York 1976, p. 149.

[21] F. Yonezawa, Prog. Theor. Phys. 40, 735 (1968).

[22] J. Tworzydło, Phys. Rev. B 50, 14591 (1994).

[23] J. Tworzydlo, Solid State Commun. 94, 821 (1995).

[24] A. Twardowski, private communication. 